\title{
Review
}

\section{Diffusional phenomena in membrane separation processes*}

\author{
G B van den Berg ${ }^{\mathrm{a}}$ and C A Smolders ${ }^{\mathrm{b}}$ \\ ${ }^{\mathrm{a}}$ Unllever Research, Process Engineering Section, Colworth House, Sharnbrook, Bedford, MK44 1LQ(UK) \\ ${ }^{\mathrm{b}}$ Dept of Chemical Engineering, University of Twente, $P O$ Box 217, 7500 AE Enschede (Netherlands)
}

(Received June 25, 1991, accepted in revised form April 6, 1992)

\begin{abstract}
Nowadays membrane filtration processes are used industrially as an alternative to conventional separation methods Membrane separation methods can be divided into classes according to their separation characteristics (1) separation by sieving action, (11) separation due to a difference in affinity and diffusivity, (111) separation due to a difference in charge of molecules, (1v) carrier-facilitated transport, and (v) the process of (time-) controlled release by diffusion In all these cases diffusion processes play an important role in the transport mechanism of the solutes Varnous mechanisms have been distinguished to describe the transport in membranes transport through bulk material (dense membranes), Knudsen diffusion in narrow pores, viscous flow in wide pores or surface diffusion along pore walls In practice, the transport can be a result of more than only one of these mechanisms For all of these mechanısms models have been derived The characteristics of a membrane, e $g$ its crystallinity or its charge, can also have major consequences for the rate of diffusion in the membrane, and hence for the flux obtained Apart from the diffusion transport processes in membranes mentioned above, other important diffusion processes are related to membrane processes, viz diffusion in the boundary layer near the membrane (concentration polarization phenomena) and diffusion during membrane formation The degree of concentration polarization is related to the magnitude of the mass transfer coefficient which, in turn, is influenced by the diffusion coefficient The effect of concentration polarization can be rather different for the various membrane processes The phase inversion membrane formation mechanism is determined to a large extent by the kinetic aspects during membrane formation, which are diffusion of solvent and of non-solvent and the kinetics of the phase separation itself
\end{abstract}

Keywords diffusion, theory, review

\section{Introduction}

\section{Membrane filtratıon processes nowadays are}

Correspondence to G B van den Berg, Unilever Research, Process Engineerıng Section, Colworth House, Sharnbrook, Bedford MK44 1LQ, UK

*Paper presented at the Int Symp "Progress in Membrane Science and Technology", Enschede, Netherlands, June 2528, 1991 used industrially as an alternative to conventional separation methods such as distillation, centrifugation and extraction. Since the first asymmetric reverse osmosis membranes became avalable in the early sixties membrane technology has developed enormously This is expressed in the vast amount of research that has gone into developing the right membrane type and module for different kinds of separa- 
tion processes, developing new processes and the best possible circumstances for separation These efforts have resulted in the present day commercialization of ultrafiltration (UF), microfiltration (MF), reverse osmosis or hyperfiltration (RO), gas separation (GS), pervaporation (PV), (kıdney-) dialysis and electrodialysis (ED)

A membrane separation process can be generally represented as in $F_{1 g} 1$

The various membrane separation methods can be divided into three classes according to their separation characteristics

(1) UF and MF use the size of the solutes to separate particles by sieving action, with a pressure difference as the driving force, the membranes used in UF can have pores from 1 to $50 \mathrm{~nm}$, while for $\mathrm{MF}$ the pore range is from 005 to $10 \mu \mathrm{m}$

(11) RO, gas separation and pervaporation, which are associated with (partly) dense membrane structures (pores $<1 \mathrm{~nm}$ ), make use of a difference in affinity between several feed components and the membrane, and of a difference in diffusivity through the membrane, the driving force is a pressure difference in case of $\mathrm{RO}$ and gas separation, and in case of dialysis and pervaporation the driving force is a concentration gradient

(iil) Electrodialysis uses anıon- and cation selective membranes to separate charged molecules from uncharged ones The ions are transported by a diffusional mechanism, as a result of an applied potential difference

Some other membrane processes are under development at the moment, for instance, facilitated transport by liquid and fixed site car-

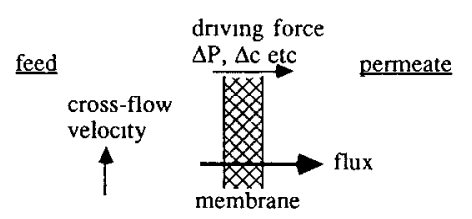

Fig 1 Schematic representation of a membrane process rier membranes Carrier-facilitated transport can also be determined by diffusion only, which depends for example on the kind of membrane applied Furthermore, the technique of controlled release of drugs from (biodegradable) reservoirs is a process that is mainly determined by diffusion

Applications of already commercialized techniques include

- Food industry: whey processing (RO and UF), concentration of milk for cheese production (UF), clarification and/or sterilization of various fluids such as wine, vinegar and apple juice (MF) and whey desalting (ED)

- Water treatment production of high resistivity (>18 M $\Omega-\mathrm{cm}$ ) water for the electronics industry ( $\mathrm{MF}$ and $\mathrm{RO}$ ) and production of clean boller feedwater, potable water and clean waste water ( $\mathrm{RO}$ and $\mathrm{ED}$ )

- Others oll-water separation (UF and MF), recovery of paint and latices from waste water effluents (UF), hemodialysis, membrane electrodialysis and recovery of gases (GS)

The membranes used in the various membrane processes can be very different, both the material and the configuration (modules) offer several possibilities A membrane can be made out of a polymeric or inorganic material Well known polymer materials are polysulfone, cellulose-acetate, polycarbonate, polypropylene and polyacrylonitrile A large variety of polymeric membranes are produced to optimize their permeability and separation characteristics. Inorganic membranes (usually MF-type) can be made from e g $\alpha-\mathrm{Al}_{2} \mathrm{O}_{3}$ or silica $\left(\mathrm{SiO}_{2}\right)$ eventually supplied with a less porous $\gamma-\mathrm{Al}_{2} \mathrm{O}_{3}$ top layer

Membranes can be subdivided in symmetrıc and asymmetric membranes Symmetric membranes can be dense, or can have straight or sponge-like pores Polymeric asymmetric membranes, usually made by the phase-inversion method, consist of a thin dense skın layer ( 0 1-1 $\mu \mathrm{m}$ thickness, containing pores or not) on top of a much more porous sublayer The thınness of the skın layer results in a low resıs- 
tance for transport through the membrane Another example of an asymmetric membrane is a composite membrane, which is usually made of a very permeable UF membrane into which a thin dense layer, often of another polymer type, is applied and which is chosen for its high selectivity

Apart from the diffusion processes mentioned above, that determine the actual membrane separation, other important diffusion processes are related to membrane processes, viz diffusion in the boundary layer near the membrane (concentration polarization phenomena) and diffusion during membrane formation: the inflow and outflow of solvents and non-solvents during the phase inversion process determine the structure of the membrane to be formed

The various diffusion processes that can occur during processes in which membranes are involved are summarized in Table 1.

The aim of this paper is to show the importance of diffusion related to membrane separation processes $A$ description of the different diffusion processes will be given in the paragraphs to come

\section{Diffusion in membranes}

When describing transport in membranes varıous possible mechanısms can be distın- guished in relation to membrane structure -transport through bulk material (dense membranes), -Knudsen diffusion, in narrow pores, -viscous flow, in wide pores, -surface diffusion, along pore walls In practice, the overall transport can be the result of more than only one of these mechanisms, this is for example the case in an asymmetric membrane, as shown in Fig 2.

It is not possible to define fixed pore-dimensions coupled to the various transport mechanisms that occur Apart from the case in which there are no pores at all (dense membranes in which sorption and diffusion of the permeants occurs ), transport will be a combination of the various mechanısms mentioned. In general, the transport can be considered to be mainly of the Knudsen diffusion type when the pore radius $r$ is smaller than $10^{-8} \mathrm{~m}(=10 \mathrm{~nm})$ at ambient pressures, and it will be mainly viscous (Poiseuille) flow when $r$ is larger than $10^{-5} \mathrm{~m}(=10$ $\mu \mathrm{m}$ ) These values also depend on the applied pressure and temperature In between these pore sizes the flow is a combination of Knudsen and Poiseuille flow [1]

\section{Transport in dense membranes}

In the literature usually one of the following three theories/models are used to describe the

\section{TABLE 1}

Survey of the various areas where diffusion occurs

1 In membranes (a) in dense membranes (gas separation, pervaporation)

(b) in porous membranes

2 In the boundary layer near the membrane

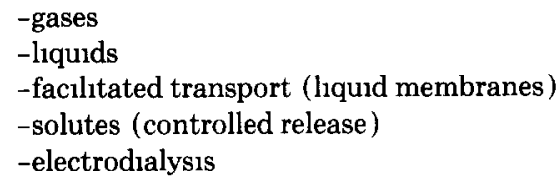

3 During membrane formation 


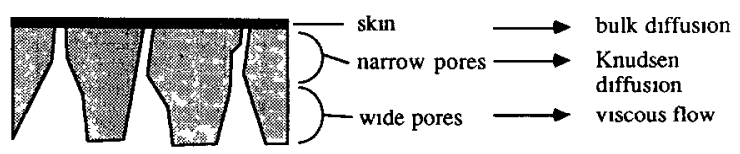

Fig 2 Transport in an asymmetruc membrane can be the result of various mechanisms

transport in dense membranes [2,3]:

I -thermodynamics of irreversible processes (TIP),

II -preferential sorption capillary flow model (PSCF),

III -solution-diffusion model (SD)

Since diffusion is the only possible transport mechanism in dense membranes a diffusion coefficient appears, directly or indirectly, in the three models mentioned above

(TIP): diffusion coefficients are incorporated via the frictional coefficients,

(PSCF) after (preferential) sorption, surface diffusion occurs at the walls of the 'pores',

(SD): the transport in the membrane is determined by both the sorption and the diffusion of the permeants

\section{Thermodynamics of irreversible}

\section{processes}

Since transport phenomena are due to nonequilibrium conditions and thermodynamics deal with equilibrium systems, the classical theory of thermodynamics is in principle less appropriate to describe transport processes We assume firstly that simultaneously occurring processes can be clearly separated into non-1nteracting mechanisms of change in the system, and secondly that the steady state irreversible flows affect only each of the processes separately At near equilibrium conditions of the irreversible processes, the thermodynamic treatment may be applied by dividing the system in small subsystems in which a local equilibrium exists or is approached.

Equations for transport rates (fluxes), retention and other membrane related parameters can be derived using general thermodynamic equations For example, the flux for each component, $J_{v}$, is described as [4]

$J_{\imath}=\sum_{k=1}^{n} L_{\imath k} F_{k}$

where the phenomenological coefficients, $L_{t}$, are often complex functions of composition and concentration The driving forces, $F_{l}$, which are chemıcal potentıal gradients, are given by

$F_{\imath}=-\left(\bar{v}_{\imath} \frac{\mathrm{d} P}{\mathrm{~d} x}+\frac{\mathrm{d} \mu_{\imath}^{\mathrm{c}}}{\mathrm{d} x}\right)$

in which the first term indicates the gradient due to a pressure difference and the second term expresses the concentration related gradients According to the friction model, the generalized force acting on solute $\imath$ in the membrane is balanced by the frictional force between solute $\imath$ and the membrane, and that between solute $\imath$ and solute $J$

$F_{\iota}=\sum f_{l \jmath}\left(u_{\imath}-u_{j}\right)+f_{\iota \mathrm{m}} u_{\imath}$

where $u_{\imath}$ and $u_{j}$ are the linear velocities of solute $\imath$ and $J$ in the membrane In practice the friction coefficients, $f_{l \mathrm{~m}}$, are always larger than the 'free friction coefficients', $f_{l}$, which are related to diffusion coefficients by

$f_{l \jmath}=R T /\left(N_{\mathrm{av}} D_{\imath \jmath}\right)$

where $D_{v}$ is the 'free' diffusion coefficient, in a 
binary solution $f_{t J}$ can be described by, e g Stokes' relation.

$f_{l \jmath}=6 \pi \eta R_{\mathrm{s}}$

in which $R_{\mathrm{s}}$ is the Stokes' radius of the solute Of course it is quite a problem to get quantitative values for the various friction coefficients, especially $f_{\mathrm{rm}}$ values

\section{The preferential sorption capillary flow} model $[5,6]$

If the pores in a dense membrane are small enough one should consider the transport phenomena taking place at the pore walls. Surface diffusion, or surface flow, can take place when the residence time of a molecule at the pore wall is larger than zero Depending on the migration energy and the surface diffusivity a molecule can move along a pore wall Because of differences in energies and diffusivities a certain selectivity in transport rate can be the result.

For component $\imath$ the flux, $N_{\mathrm{S}}$, due to surface diffusion can be described as.

$N_{\mathrm{S}_{\imath}}=-C^{\prime} D_{\mathrm{S}_{\imath}}\left(\mathrm{d} C_{\mathrm{S}_{\imath}} / \mathrm{d} p\right) \nabla P$

where $C^{\prime}$ is a geometrical parameter that depends on the pore system, and $\left(\mathrm{d}_{\mathrm{S}_{l}} / \mathrm{dp}\right)$ can be determined from the adsorption isotherm The surface diffusion coefficient $D_{\mathrm{S} \imath}$ is a function of the temperature as is given by

$D_{\mathrm{S}_{\iota}}=D_{\mathrm{S} 0_{2}} \exp (-E / R T)$

while $D_{\mathrm{SO} \text { s }}$ depends on the mean surface freepath length, the jump frequency and the surface concentration (especially at low surface concentrations) The activation energy for surface diffusion (migration energy) $E$ is assumed to be a fraction of the differential heat of adsorption $q$

$E=q / m^{\prime}$

where $m^{\prime}=1,2$ or 3 , which depends on the type of surface binding. Now it can be derived that
$N_{\mathrm{S} \imath}=-C^{\prime} D_{\mathrm{S} 0 \imath} \exp \left(-q / m^{\prime} R T\right)\left(\mathrm{d} C_{\mathrm{S}_{\imath}} / \mathrm{d} p\right) \nabla P$

From experiments it appeared that the surface diffusion coefficients can be described very generally without taking the influence of the surface concentration into consideration as [6]:

$D_{\mathrm{S}}=16 \times 10^{-6} \exp \left(-045 q / m^{\prime} R T\right)$

Though the existence of pores seems to be contradictory to the tightness of dense membranes, this surface diffusion model can be used to describe transport in dense membranes' the intermolecular voids are considered to be the 'pores' For the description of transport in RO membranes the situation is thought to be as described in Fig 3 [7]

Furthermore, the surface diffusion approach can also be used in combination with other models For instance, Jonsson et al [8] described the flux as a result of a combination of diffusional surface flow and viscous flow through the 'pores' of a RO membrane

\section{The solution-diffusion model}

This model, which in practice is most widely used for dense membranes, describes the transport by permeablity $=$ sorption $\times$ diffusion [9] This can be seen in the general expression for the flux $J_{\imath}$

$J_{\imath}=-D_{\imath} c_{\imath}^{\mathrm{m}} \mathrm{d}\left(\mu_{\imath}^{\mathrm{m}} / R T\right) / \mathrm{d} x$
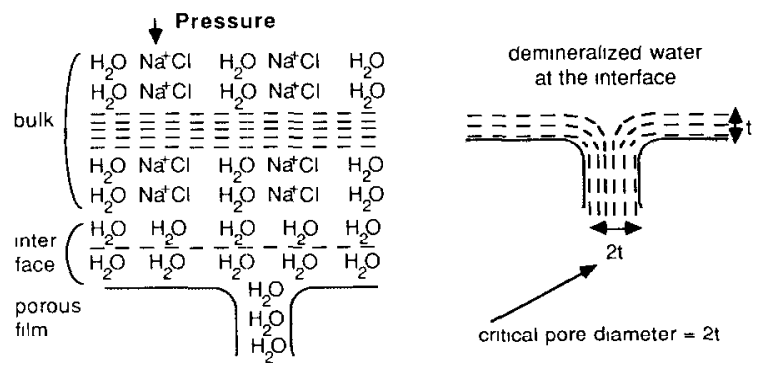

Fig 3 Schematical representation of the preferential sorption capillary flow model The dimension of the critical pore diameter is two times the thickness of the adsorbed water layer 
where the gradient in the chemical potentıal across the membrane $\mathrm{d}\left(\mu_{\imath}^{\mathrm{m}}\right) / \mathrm{d} x$ is the driving force The concentration $c_{l}^{\mathrm{m}}$ in the membrane can be calculated using, e $\mathrm{g}$, the Flory-Huggins equation

In the absence of strong interaction effects between the permeating components, the permeability constants $P$ of the pure components can be used to describe the selectivity

$\alpha_{\mathrm{A} / \mathrm{B}}=P_{\mathrm{A}} / P_{\mathrm{B}}=\left(S_{\mathrm{A}} / S_{\mathrm{B}}\right) \times\left(D_{\mathrm{A}} / D_{\mathrm{B}}\right)$

where the preferential sorption is expressed by $S_{\mathrm{A}} / S_{\mathrm{B}}$ and the ratio of the mobilities by $D_{\mathrm{A}} /$ $D_{\mathrm{B}}$.

Both the sorption and the diffusion are dependent on the characteristics of the membrane material and the permeants In the literature many examples can be found attempting to describe the permeation selectıvity as a function of the sorption and/or the diffusion coefficient. Both in experimental and theoretıcal papers the solution-diffusion model has been used For instance, Lee et al [10] showed that, during pervaporation of organics from dilute aqueous solutions, the product of independently measured distribution (sorption ratio) and diffusion coefficients correlated reasonably well with the measured permeability coefficients.

On the other hand, also more basic theoretical descriptions have been given, as can be found below For both sorption and diffusivity a number of (modified/1mproved) relations have been derived, this is quite understandable because of the many assumptions that had to be made to derive the most simple equations In this survey only a limited number of relevant equations will be given

(a) The sorption of the permeants in the polymer matrix can be described by, for instance, the Flory-Huggins equation because a swollen membrane may be considered to be a homogeneous liquid mixture of polymer and permeants' the partial molar free energy of mixing of a solvent and a polymer is [2]

$F_{\mathrm{s}}^{\mathrm{mix}}=R T\left[\ln \Phi_{\mathrm{s}}+(1-1 / \mathrm{m}) \Phi_{\mathrm{p}}+\chi \Phi_{\mathrm{p}}^{2}\right]$

where $\chi$ is the Huggins' parameter, $m$ is the molar volume ratio of polymer and solvent, and $\Phi_{\mathrm{p}}$ and $\Phi_{\mathrm{s}}$ are the volume fractions of the polymer and the solvent, respectively, from which the concentration $c_{t}^{\mathrm{m}}$ can be calculated. Huggins' parameter $\chi$ depends on the solubility parameters of solvent and polymer, among other things

In case of gas separation a different model is frequently used the dual-mode sorption model This model combines Henry's law with a Langmuir isotherm The latter reflecting the sorption of a gas in voids that are assumed to exist in a glassy polymer membrane For comparison, the solubilities of gases in rubbery polymers above the $T_{\mathrm{g}}$ often are described by Henry's law only The resulting descriptions of the sorption equilıbria are [11]

$$
\begin{array}{ll}
T>T_{\mathrm{g}} \cdot & C=k_{\mathrm{D}} p \\
Y<T_{\mathrm{g}} & C=k_{\mathrm{D}} p+\left(C_{\mathrm{H}}^{\prime} b p\right) /(1+b p)
\end{array}
$$

where $k_{\mathrm{D}}$ is Henry's law solubility coefficient, $C_{H}^{\prime}$ is the Langmuir capacity constant and $b$ is an affinity constant

(b) The diffusive transport can be described by the (modified) Maxwell-Stefan (M-S) equation, whereas the (modified) Vigne equation can be used to calculate the necessary mixture diffusivities The M-S equation

$\frac{c_{\imath} \operatorname{grad}\left(\mu_{\imath}\right)}{R T}=\sum_{\substack{\jmath=1 \\ j \neq \imath}}^{n} \frac{x_{\imath} N_{\jmath}-x_{\jmath} N_{\imath}}{D_{\imath}}$

uses the binary M-S diffusivities, $D_{\imath}$, of $\imath$ in a mixture of $\imath$ and $\jmath$, which are not equal to the Fickian diffusion coefficients (though in binary gases and dilute solutions they are about equal). It has been observed that these diffusivities vary considerably with composition and concentration, although much less than Fickian diffusion coefficients do Therefore a mod- 
ified $\mathbf{M}-\mathrm{S}$ equation (MM-S) has been derived, no longer assuming a frictional force that is independent of concentration and composition [12]

$$
\frac{c_{\imath} \operatorname{grad}\left(\mu_{\imath}\right)}{R T} * D_{\imath \mathrm{m}}=\sum_{\substack{\jmath=1 \\ j \neq \imath}}^{n}\left(x_{\imath} N_{\jmath}-x_{\mathrm{j}} N_{\imath}\right)
$$

The MM-S equation uses the coefficients of self diffusion, ${ }^{*} D_{l \mathrm{~m}}$, of a species $l$ in a mixture In all the equations mentioned above the membrane can be considered to be one of the components of the mixture The self diffusion coefficients, ${ }^{*} D$, in a multicomponent mixture can be described by a Vigne equation, which read originally $\ln \left(D_{l}\right)=x_{\imath} \ln \left({ }^{\infty} D_{\jmath \iota}\right)+x_{\jmath} \ln \left({ }^{\infty} D_{\jmath}\right)$, modified to

$$
\ln \left({ }^{*} D_{\imath \mathrm{m}}\right)=x_{\imath} \ln \left({ }^{*} D_{u l}\right)+\sum_{\substack{j=1 \\ J \neq \imath}}^{n} x_{j} \ln \left({ }^{\infty} D_{\imath \jmath}\right)
$$

where ${ }^{\infty} D_{l \jmath}$ is the diffusivity of $l \mathrm{in} J$ at infinite dilution of $\imath$.

The crystallınity of a membrane can also have major consequences for the rate of diffusion in the membrane, and hence for the flux The diffusion coefficient as a function of the crystallinity can be described as follows:

$D_{\imath}=D_{\iota, 0} \frac{\Psi_{c}^{n}}{B}$

where $\Psi_{\mathrm{c}}$ is the fraction crystalline polymer, B is a constant and the exponent $n \leqslant(\ll) 1$ The effect can be a hundredfold increase in diffusion resistance, $\mathrm{F}_{1 \mathrm{~g}} 4$ [12]

The influence of the molecular weight (hence size) of the components on the diffusion is shown in Fig 5 To emphasize the influence of the rubbery/glassy state of the polymer the diffusion in water, natural rubber (rubbery state) and polystyrene (glassy state) are given [13] The drawn lines have been obtained from a large number of experimental data. The difference in value for the diffusion coefficient in

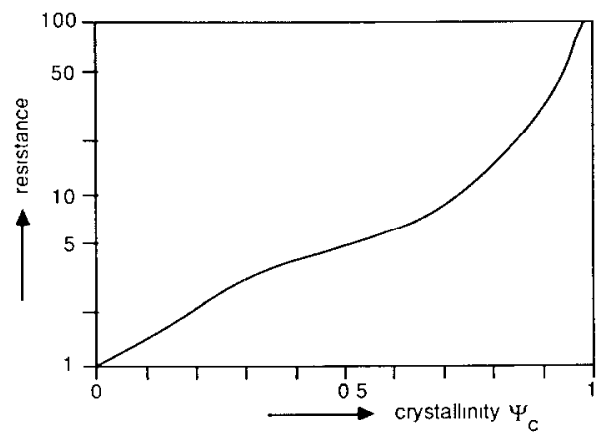

Fig 4 Possible effect of crystallinity on diffusion resistance Taken from Ref [12]

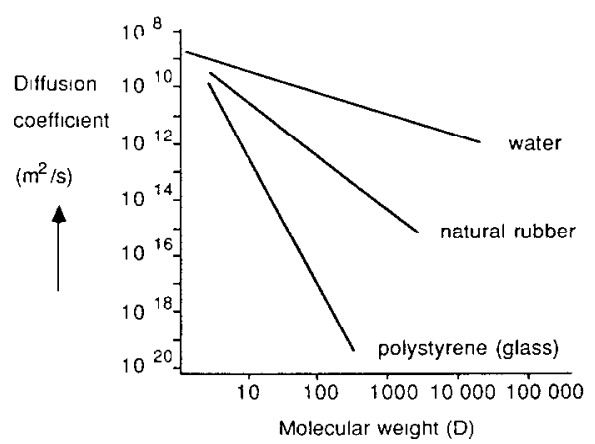

Fig 5 Diffusion coefficients, in water, in natural rubber and in glassy polystyrene, as a function of the molecular weight of the diffusing particle

these different media is shown to be several orders of magnitude for an average size molecule

When selectively permeable materials are Incorporated in a dense membrane, both the selectivity and the flux can be positively changed. Cussler [14] showed that in flake-filled membranes the selectivity and the relative flux can be greatly enhanced An optımum ratio of the diffusion coefficient in the polymer and the diffusion coefficient in the flakes can be calculated using variable flake fractions in the membrane and variable flake-width/thıckness ratios

\subsection{Transport in porous membranes}

As stated in the introduction transport through bulk material (dense membranes), will 
be different from the transport phenomena in more porous membranes Knudsen diffusion in narrow pores and viscous flow in wide pores are more likely to occur in porous membranes The various possibilities to consider are transport phenomena of gases, solutes and liquids, facilitated transport, solutes in controlled release and electrodialysis These subjects will be described in the next paragraphs

\section{Knudsen diffusion in narrow pores}

Knudsen diffusion occurs when the pore diameter is much smaller than the mean free path lengths, $\lambda$, of the (gas) molecules to be separated. $\lambda$ is defined as:

$\lambda=\frac{3 \eta}{\rho_{\mathrm{g}}} \sqrt{\frac{\pi M_{\mathrm{A}}}{8 R T}}$

where the viscosity $\eta$ and the density $\rho$ are functions of $T$ and $\Delta P$ The flux due to Knudsen diffusion in straight pores can be described by

$J_{\mathrm{A}}=\frac{4 r}{\sqrt{2 \pi k T}} \frac{1}{\sqrt{M_{\mathrm{A}}}} \frac{\mathrm{d} P}{\mathrm{dx}}$

or by

$J_{\mathrm{A}}=\frac{2}{3} \pi n_{\mathbf{k}} r^{3} \frac{\Delta P}{\delta} \frac{\overline{v_{\mathrm{A}}}}{R T}$

$\overline{v_{\mathrm{A}}}$ is the average molecular velocity; it is normally represented by.

$\overline{v_{\mathrm{A}}}=\sqrt{\left(8 R T / \pi M_{\mathrm{A}}\right)}$

From the first flux equation the selectivity $\alpha$ can be derived, as

$\alpha_{\mathrm{A} / \mathrm{B}}=\sqrt{\left(M_{\mathrm{B}} / M_{\mathrm{A}}\right)}$

The second equation shows the relation to diffusion. The Knudsen diffusion coefficient in a pore with diameter $d_{\mathrm{p}}$, or radius $r$, can be calculated as

$D_{\mathrm{KA}}=\frac{1}{3} \overline{\mathrm{v}_{\mathrm{A}}} d_{\mathrm{p}}\left(\frac{2-f}{f}\right)$ (in which $f$ is the fraction diffuse reflection which the pore wall); this is roughly equal to $\frac{2}{3} \overline{v_{\mathrm{A}}} r$ when $f$ equals about unity

The importance of Knudsen diffusion relative to molecular diffusion, which is given by the number of collisions with the wall compared to the number of mutual collisions, can be described for hard spheres of pure component $A$ as $\lceil 15\rceil$.

$\frac{D_{\mathrm{KA}}}{D_{\mathrm{AA}}}=\frac{d_{\mathrm{p}}}{\lambda}$

For example, for $\mathrm{N}_{2}$ at a pressure of $01 \mathrm{MPa}$ and pores of $d_{\mathrm{p}}=5 \mathrm{~nm}$, more than $90 \%$ of the collisions will be collisions with the wall and so Knudsen diffusion mainly determines the transport

The importance of surface diffusion relative to Knudsen diffusion has been described in literature as well Keizer et al. [1] found that the observed permeability of $\mathrm{CO}_{2}$ was larger than could be calculated from the permeability of $\mathrm{N}_{2}$ and ascribed the additional transport to surface diffusion (Fig. 6).

Uhlhorn et al [16], using silica modified $\gamma$ alumina membranes, found much higher selectivities for condensable gases than Knudsen diffusion could account for The modification of the $\gamma$-alumina membranes with silica con-

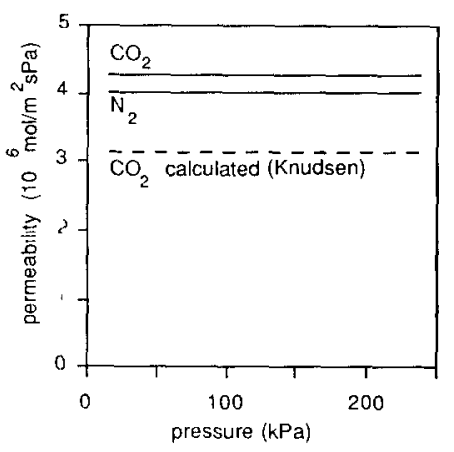

Fig 6 The permeabilities of $\mathrm{CO}_{2}$ and $\mathrm{N}_{2}$ as a function of pressure for the separation (top-) layer of ceramic membranes [1] The dashed line is the expected value for $\mathrm{CO}_{2}$ if only Knudsen diffusion occurs 
sisted of a sılica layer of approxımately $30 \mathrm{~nm}$ thickness, with pores smaller than $1 \mathrm{~nm}$, on top of the membrane and probably some filling of the pores with silica as well. From permeability experiments it could be concluded that transport of inert gases, e.g helium and nitrogen, was determined by Knudsen diffusion only However, for condensable gases the transport was greatly enhanced, the permeabilities were dependent on the applied pressure and surprisingly high permselectivities could be observed These phenomena were attributed to surface diffusion.

In a further study a $\gamma$-alumina membrane was impregnated with magnesia, introducing more strong-base sites and fewer weak-base sites This resulted in stronger bonding of $\mathrm{CO}_{2}$, at about equal amounts, and less surface diffusion (the surface diffusion accounts for approximately $30 \%$ of the $\mathrm{CO}_{2}$ transport on unmodified $\gamma$-alumina membranes, see Fig 6) [17]

\section{Viscous flow in wide pores}

The average velocity of a liquid medium in a pore is described by the Hagen-Poiseulle relation:

$$
\langle v\rangle=\frac{\Delta P}{32 \delta} \frac{d_{\mathrm{p}}^{2}}{\eta}
$$

from which the flux $J$ can be calculated.

$J=\frac{n_{\mathrm{k}} A_{\text {pore }}}{A_{\text {membrane }}}\langle v\rangle=\epsilon\langle v\rangle=$ constant $\times r^{4}$

In this equation no molecule-related quantities are left, from which it can be easily concluded that Poiseulle flow will not result in any selectivity. Furthermore, it can be observed that now the flux is proportional to $r^{4}$, which was $r^{3}$ for Knudsen flow. So in a matrix consisting of very small pores few large pores can have great $1 \mathrm{~m}$ pact on $\alpha$

The afore-mentioned transport of combined Knudsen and Poiseulle flow has been described by Schofield et al [18] They described the flux in the Knudsen-Poiseulle transition range by

$J=a\left(P / P_{\text {ref }}\right)^{g} \Delta P$

where $a$ is the membrane constant and $g$ is a measure of the extent of the Poiseulle flow The parameter $g$ increases with increasing molecular weight of the gas and with increasing membrane pore size

Until now the examples on the performance of the membrane were mainly determined by the pore size of the membrane Obviously, the nature of the membrane also determines the separation characteristics An example is the description of a diffusion-reaction in a multımembrane containıng urease The equilibrium reaction $\mathrm{NH}_{2} \mathrm{CONH}_{2}+2 \mathrm{H}_{2} \mathrm{O} \leftrightarrow 2 \mathrm{NH}_{4}^{+}+$ $\mathrm{CO}_{3}^{2-}$ is strongly affected when one of the membranes in the cell is switched from a neutral cellulosic membrane to an anion-exchange membrane. The outward transport of the reaction products and so the conversion are greatly reduced because of the nature of the membrane [19]

\section{Facrlitated transport in liquid and} fixed site carrier membranes

Liquid membranes (LM) were developed because of the relatively small transmembrane flux of polymer membranes A liquid membrane is a fluid or quasi-fluid phase which separates two other phases that are immiscible with the liquid membrane Various types of liquid membranes exist, e g bulk-, emulsıon- and supported liquid membranes.

Because of the nature of the faclitated transport, in which a carrier is used for the selective transport of e g. an ion, the transport in the liquid membrane is mainly determined by the diffusion of the carrier in the membrane phase Transport of the ion can even take place against its own concentration gradient. This is possible because of the phenomenon of coupled transport, 1.e a different ion of equal charge is 


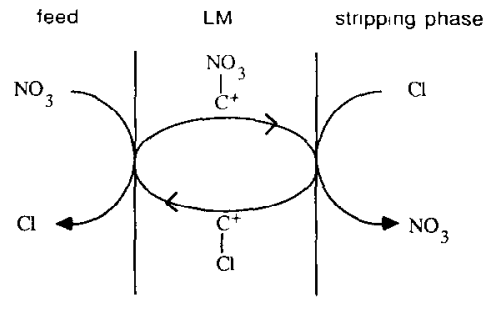

Fig 7 The (supported liquid membrane) separation with a countertransport mechanism for the removal of nitrate lons

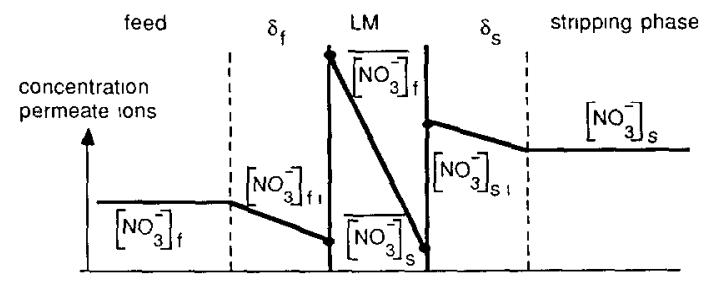

Fig 8 Ideal concentration profile for transport of permeating $\mathrm{NO}_{3}$-1ons through a liquid membrane $\mathrm{NO}_{3}$ stands for the carrier-ion complex

transported back This ion can be provided with a large chemical potential gradient Mostly catIons are transported although also anıon transport has been realized, e $\mathrm{g}$ the removal of $\mathrm{n} 1-$ trates from ground water [20] (F1g. 7) In this process the following steps of the permeate ion can be observed.

(a) diffusion of the ion to the membrane surface,

(b) chemical reaction (complexation) at the surface with a carrier molecule,

(c) diffusion of the carrier bounded ion through the LM-phase,

(d) chemical reaction at the surface with the stripping phase (decomplexation),

(e) diffusion of the ion to the bulk of the strupping phase

The expected concentration profile of the ion is shown in Fig 8 In the most simple representation of the process the flux of the anion can be represented by Fick's law like $J=\Delta C D / \delta$ and so it will be a linear function of the diffusion coefficient of the complex in the LM The dif- fusion coefficient of the carrier-anion complex is usually calculated using the Stokes-Einstein equation. $D=k T /\left(6 \pi \eta_{0} r\right)$. Other diffusivities can play a role as well; e $g$ the diffusion of the anion in the stagnant film at both sides of the membrane or the back-diffusion of the carrier However, in case of nitrate removal the simple Fick's law can be applied

Noble [21] developed a model for transport in fixed-site carrier membranes (the complexing agents are cast directly into the polymer films ) This model yields a dual-mode sorption kind of description in the case of diffusion-limited transport The diffusion in this case is determined by the diffusion coefficient of the solute-carrier complex This implies that the transport is morphology dependent Furthermore, it was shown that the change in mobility caused by changes in morphology may result in a percolation limit

\section{Solute transport in controlled release} (CR) [22]

The release of a drug (e $g$ the contraceptive sterold levenorgestrel) from a CR-membrane, which is usually a microporous hollow fibre membrane, can be described in two ways depending on the tightness of the skin

(a) One way of release is described as a solution-diffusion mechanism, $1 \mathrm{e}$ the steroid dissolves into the (dense) polymeric skin, diffuses through the polymer and finally partition occurs into the recelving fluid surrounding the hollow fibre On the assumption that drug dissolution is not rate-limiting for such a mechanism two extreme conditions may exist. (1) the rate-controlling step is formed by diffusion of the steroid through the hollow fibre skın (membrane-controlled permeation process), or (11) by diffusion of the steroid from the hollow fibre into the receiving fluid (diffusion layerlimiting partition-controlled process)

In case (1) the transport is proportional to the diffusion coefficient of the steroid in the 
polymeric skın $D_{\mathrm{sp}}$, proportional to the concentration difference between the concentration in and outside of the fibre and inversely proportional to the thickness of the skin layer:

$\mathrm{d} M_{t} / \mathrm{d} t=k_{1} D_{\mathrm{sp}} A C / d_{\mathrm{m}}$

In case (11) (diffusion layer-limiting part1tion-controlled process) the transport is proportional to the diffusion coefficient $D_{\mathrm{sr}}$ and the solubility $C_{\mathrm{r}}$ in the receiving fluid, and inversely proportional to the thickness of the diffusion boundary layer $d_{\mathrm{h}}$.

$\mathrm{d} M_{t} / \mathrm{d} t=k_{2} D_{\mathrm{sr}} C_{\mathrm{r}} / d_{\mathrm{h}}$

(b) When the asymmetric (hollow fibre) membrane contains a microporous skin a porediffusion mechanism can be imagined The steroid release rate, into the liquid-filled pores, is then described as follows

$\mathrm{d} M_{t} / \mathrm{d} t=k_{2} D_{\mathrm{sr}}(\epsilon / \tau) C_{\mathrm{r}} / d_{\mathrm{m}}$

where $d_{\mathrm{m}}$ is the thickness of the microporous skin, and $\epsilon$ and $\tau$ are the porosity and the tortuosity of the membrane wall, respectively

\section{Electrodialysıs [23]}

The separation of ions by electrically charged membranes under the influence of the driving force $\Delta E$ is schematically represented in Fig 9

The transport during electrodialysis is determined by two processes in which diffusion plays an important role

(a) the transport through the membranes that causes the actual separation of anions and cat-

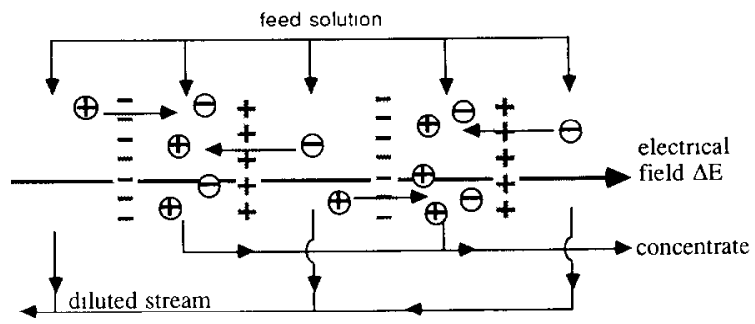

Fig 9 Schematic representation of the electrodialysis membrane separation process 10ns. The diffusion involved is diffusion of ions in swollen polymers, and

(b) the transport through the inevitable concentration polarization boundary layer In this case the diffusion is diffusion of an ion in the solvent

Usually concentration polarization phenomena influence the electrodialysis process to a large extent, which results in the observation that the transport is mainly determined by the diffusion process of the ions in the boundary layers near the membrane For instance, Tanaka [24] has shown that ion-exchange electrodialysis of $\mathrm{NaCl}$ results in concentration polar1zation boundary layers on both the anion and the cation exchange membranes Due to the diffusion coefficient of $\mathrm{Cl}^{-}$-1ons being larger than the diffusion coefficient of the $\mathrm{Na}^{+}-10 \mathrm{~ns}$, the concentration near the cation exchange membrane will rise considerably As can be expected the concentration near the anion exchange membrane will drop compared to the bulk concentration However, this is only the case when the convective flow rate towards the membrane stays below a critical value, if not, a concentration equal to the bulk concentration can be expected

In practice, the concentration polarization problem can be reduced considerably by introducing the electrodialysis reversal mode in which the polarity of the electrodes is reversed periodically, typically every $15 \mathrm{~min}$ [25]

\section{Diffusion in the boundary layer near the membrane (concentration polarization related phenomena) [26]}

'The description of concentration polarization phenomena during membrane separation processes can be generally divided into two groups (F1g 10).

-cake filtration, which is used for membrane processes that involve large particles, like in $\mathrm{MF}$ and sometimes UF, and 


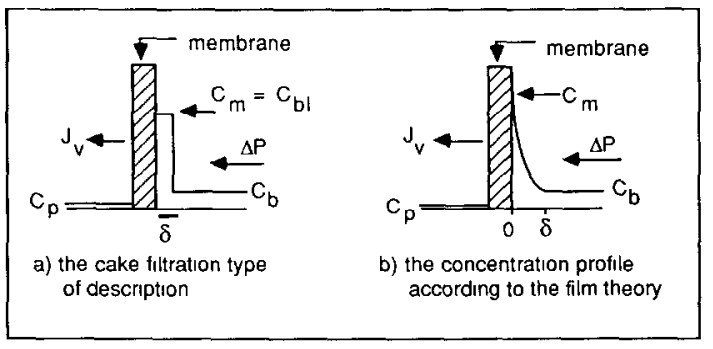

Fig 10 The concentration profiles due to concentration polarization phenomena according to the cake-filtration type of description (a) and the film theory (b)

-film theory description, which is used for all processes that separate liquids, 1ons and small partıcles. Examples are pervaporation (L-L), FD (1ons), RO (small molecules) and often UF (macromolecules)

In most concentration polarization equations a flux expression like $J=k \times f\left(C_{\mathrm{b}}, C_{\mathrm{p}}, \quad\right)$ is used, e $g$ the well known equation

$J_{\mathrm{v}}=k \ln \left\{\left(C_{\mathrm{m}}-C_{\mathrm{p}}\right) /\left(C_{\mathrm{b}}-C_{\mathrm{p}}\right)\right\}$

where $k$ is the mass transfer coefficient, and $k=D / \delta$, in which $\delta$ is the thickness of the boundary layer The diffusion coefficient of the solute in the solution that has to be used in this equation is usually taken to be independent of concentration However, some researchers have shown that a concentration dependent diffusion coefficient can describe the process much better [27] The effect of concentration polarization can be rather different for the various processes (see Table 2)

For ultrafiltration a number of different models have been described in literature, both for dead-end and for crossflow filtration $\mathrm{A}$ model that appeared to be able to predict fluxes accurately during dead-end ultrafiltration of proteins is the Boundary Layer Resistance model [28,29] Mainly using characteristics of the proteins, like sedimentation coefficient and specific volume, the flux behaviour could be calculated numerically For instance, the concentration profiles after various periods of fil-
TABLE 2

The influence of concentration polarization on various membrane separation processes

\begin{tabular}{lll}
\hline Process & $\begin{array}{l}\text { Influence of } \\
\text { concentration } \\
\text { polarization }\end{array}$ & Reason \\
\hline Reverse osmosis & moderate & $k$ large \\
Ultrafiltration & strong & $k$ small \\
Microfiltration & strong & $k$ small \\
Gas separation & (very) low & $k$ very large $/ J$ small \\
Pervaporation & low & $k$ large $/ J$ small \\
Dialysis & low & $J$ small \\
Electrodialysis & strong & \\
\hline
\end{tabular}

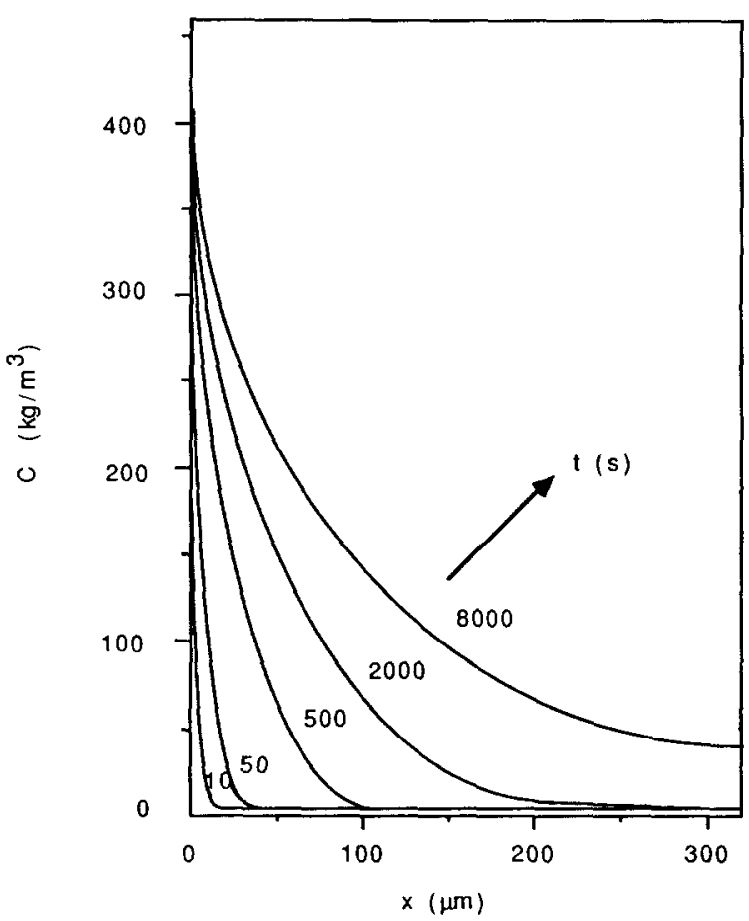

Fig 11 Simulated concentration profiles near the membrane interface for ultrafiltration of the protein BSA, as a function of time and distance from the membrane, using $D=69 \times 10^{-11} \mathrm{~m}^{2} / \mathrm{sec}$

tration of a BSA solution are reproduced in Fig 11 [29]

The influence of the value of the diffusion coefficient on the build-up of the concentration profile was also calculated (Fig 12). The figure 


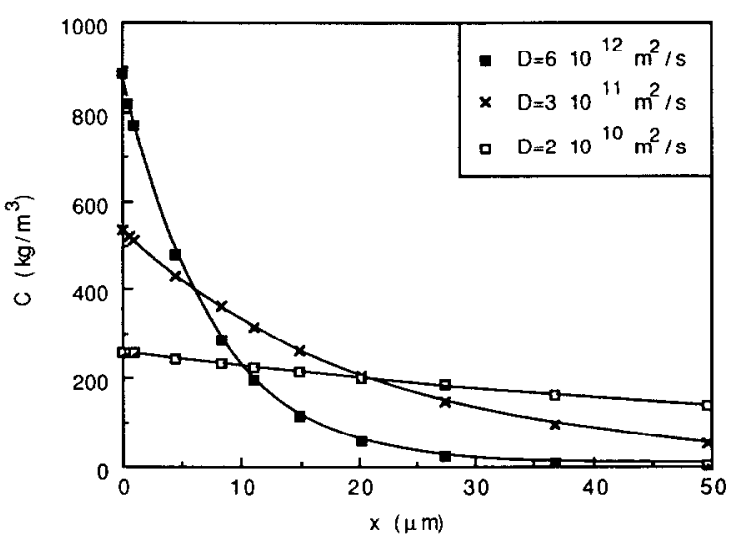

Fig 12 The calculated concentration profiles after 1000 sec ultrafiltration of BSA using various values of the diffusion coefficient

clearly shows the increasing concentration near the membrane with decreasing diffusion coefficient, this phenomenon will result in an increased resistance of the concentrated boundary layer near the membrane, while the separation characteristics are also affected strongly

\section{Diffusion during membrane formation [30]}

The most important preparation technique for polymeric membranes is the phase inversion method. This method results in various types of membranes by precipitation from a polymer solution using a non-solvent that diffuses into a film of polymer solution. Both symmetric and asymmetric membranes can be formed, with porous or dense top layers, depending on the membrane formation mechanısm. Frequently used polymers are polysulfone, polyacrylonitrile, polypropylene, cellulose-acetate and polyamide.

The preparation technique is based on precipitation after immersing the polymer solution in a non-solvent bath, resulting in the name 'immersion precipitation' Instead of immersing the polymer solution in pure non-solvent, a mixture of solvent and non-solvent can also be used as the coagulation bath

The membrane formation mechanism by phase separation is determined by. (a) thermodynamic equilibria in the polymer/solvent/ non-solvent system, and (b) the kinetıc aspects during membrane formation, which are (b1) diffusion of solvent (out) and non-solvent (into the polymer film) and (b2) kinetics of the phase separation itself Usually the phase behaviour of the three component system polymer/solvent/non-solvent is represented in a phase diagram The changing compositıon during membrane formation will determine which type of membrane will be developed. Two possible composition paths (leading to either aggregate formation or L-L demixing) during coagulation determine the type of membrane that will be formed These diffusion determined processes are represented in the phase diagram (Fig. 13).

The calculation of the composition profile is done using (a) ternary diffusion equations, (b) the appropriate boundary conditions, and (c) concentration dependent diffusion coefficlents. The fluxes of the various components are depicted in Fig 14

The ternary diffusion equations are for the polymer solution:

$$
\begin{gathered}
\partial\left(\phi_{\imath} / \phi_{3}\right) / \partial t=\partial / \partial t\left[v_{\imath} \phi_{\imath} \sum L_{\imath \jmath}\left(\partial \mu_{\jmath} / \partial m\right)\right] \\
\quad(\iota=1,2,3)
\end{gathered}
$$

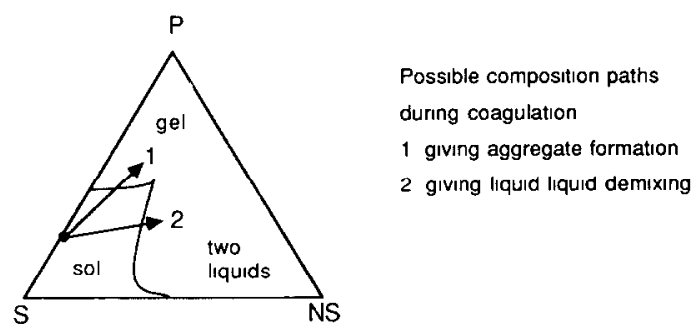

Fig 13 Phase diagram of the three component system solvent (S)/non-solvent (NS)/polymer (P) and the possible composition paths during coagulation 


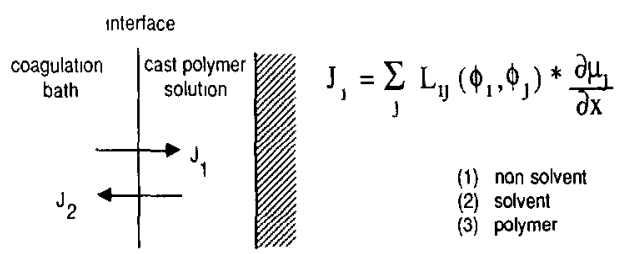

Fig 14 The situation during membrane formation

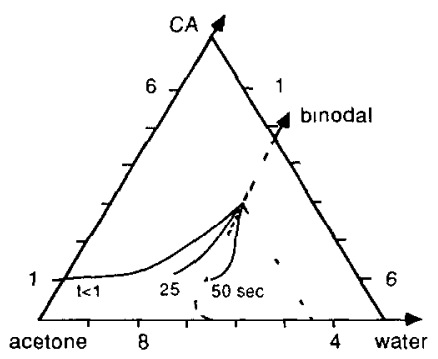

Fig 15 Approximated change of the composition path at different immersion times for a 10 vol \% cellulose acetate (CA) solution immersed into a pure water bath

and in the coagulation bath

$\partial \phi_{l} / \partial t=\partial / \partial t\left[D\left(\phi_{l}\right) \partial \phi_{\imath} / \partial x\right] \quad(l=1,2)$

The phenomenological coefficients $L_{l j}$ can be obtained from solvent-non-solvent diffusion coefficients and the sedimentation coefficient of polymer in solvent.

Different experimental circumstances can be simulated also in Fig 15 the influence of the time of immersion in the coagulation bath on the composition path is represented [30]. From the figure it will be clear that in this case a certain time is needed before liquid-liquid demixing will occur During this delay-time a dense surface layer is formed by loss of solvent from the polymer solution, resulting in an asymmetric membrane structure Consequently the k1netics of demixing determine the type of membrane that is formed

\section{Conclusions}

Diffusion processes play an important role in membrane separation processes apart from the diffusional transport processes in membranes, diffusion in the boundary layer near the membrane (concentration polarization phenomena) and diffusion during membrane formation are of high interest The different diffusion processes are described by one of the numerous models

Varıous mechanısms have been distınguished to describe the transport in membranes' transport through bulkmaterial (dense membranes ), Knudsen diffusion in narrow pores, viscous flow in wide pores, or surface diffusion along pore walls In practice, the transport can be a result of more than only one of these mechanisms.

The characteristics of a membrane, e.g its crystallinity or its charge, can also have major consequences for the rate of diffusion in the membrane, and hence for the flux obtained.

The effect of concentration polarization can be rather different for the various processes, which is mainly determined by the mass transfer coefficient, and thus by the diffusion coefficient.

The phase inversion membrane formation mechanism is determined to a large extent by the diffusion of solvent and of non-solvent

\section{List of symbols}

a membrane permeability constant $\left(\mathrm{kg} / \mathrm{m}^{2}\right.$-sec-Pa $)$

A surface area $\left(\mathrm{m}^{2}\right)$

b affinity constant (1/atm)

$B \quad$ constant (-)

$c_{l} \quad$ concentration of $\imath\left(\mathrm{kg} / \mathrm{m}^{3}\right)$

C amount of sorbed gas per amount of polymer $\left[\mathrm{m}^{3}(\mathrm{STP}) / \mathrm{m}^{3}\right]$

$C^{\prime} \quad$ geometrical parameter (-)

$C_{\mathrm{b}} \quad$ concentration in the bulk $\left(\mathrm{kg} / \mathrm{m}^{3}\right)$

$\mathrm{C}_{\mathbf{H}}^{\prime} \quad$ Langmuir capacity constant $\left(\mathrm{m}^{3}(\mathrm{STP}) / \mathrm{m}^{3}\right)$

$C_{\mathrm{p}} \quad$ concentration in the permeate $(\mathrm{kg} /$ $\mathrm{m}^{3}$ )

$C_{\mathrm{r}} \quad$ solubility in receiving fluid $\left(\mathrm{kg} / \mathrm{m}^{3}\right)$ 
$C_{\mathrm{S} \imath} \quad$ concentration of $\imath$ at the surface $(\mathrm{kg} /$ $\mathrm{m}^{3}$ )

$d_{\mathrm{h}} \quad$ thickness of the diffusion boundary layer $(\mathrm{m})$

$d_{\mathrm{p}} \quad$ pore diameter $(\mathrm{m})$

$D_{l j} \quad$ diffusion coefficient of $\imath$ in $J\left(\mathrm{~m}^{2} / \mathrm{sec}\right)$

${ }^{\infty} D_{\imath \jmath} \quad$ diffusivity of $\imath \mathrm{in} \jmath$ at infinite dilution of $l\left(\mathrm{~m}^{2} / \mathrm{sec}\right)$

${ }^{*} D_{l \mathrm{~m}} \quad$ coefficient of self diffusion of $\imath$ in a mixture $\left(\mathrm{m}^{2} / \mathrm{sec}\right)$

$D_{\mathrm{S} \imath} \quad$ surface diffusion coefficient $\left(\mathrm{m}^{2} / \mathrm{sec}\right)$

$D_{\mathrm{sp}} \quad$ diffusion coefficient of steroid in the polymerıc skın $\left(\mathrm{m}^{2} / \mathrm{sec}\right)$

$D_{\mathrm{sr}} \quad$ diffusion coefficient of sterold in receiving liquid $\left(\mathrm{m}^{2} / \mathrm{sec}\right)$

$E \quad$ activation energy for surface diffusion $(\mathrm{J} / \mathrm{mol})$

$\Delta E \quad$ potential difference (V)

$f$ fraction diffuse reflection at the pore wall (-)

$f_{l j} \quad$ friction coefficient $\left(\mathrm{J}-\mathrm{sec} / \mathrm{m}^{2}\right)$

$F_{l} \quad$ driving force (N)

$F_{l}^{\text {mux }} \quad$ partial molar free energy of mixing $(\mathrm{J} / \mathrm{mol})$

$g \quad$ exponent in Schofield's equation (-)

$J_{l} \quad$ flux of component $\imath(\mathrm{m} / \mathrm{sec})$

$J_{\mathrm{v}} \quad$ volume flux $(\mathrm{m} / \mathrm{sec})$

$k$ mass transfer coefficient $(\mathrm{m} / \mathrm{sec})$

$k_{\mathrm{D}} \quad$ Henry's law solubility coefficient [ $\mathrm{m}^{3}$ (STP) $/ \mathrm{m}^{3}$-atm]

$k_{\mathrm{n}} \quad$ constant $\left(\mathrm{m}^{2}\right)$

$L_{i j} \quad$ phenomenological coefficient $(\mathrm{kg}-$ $\mathrm{sec} / \mathrm{m}$ )

$m \quad$ molar volume ratio of polymer and solvent $(\mathrm{m})$

$m^{\prime} \quad$ factor depending on type of binding $(-)$

$M_{\mathrm{A}} \quad$ molecular weight $(\mathrm{kg} / \mathrm{kmol})$

$M_{t} \quad$ mass (kg)

$n \quad$ exponent (-)

$n_{\mathrm{k}} \quad$ number of pores (-)

$N_{\mathrm{av}} \quad$ Avogadro's number $(1 / \mathrm{mol})$

$N_{\imath} \quad$ molar flux $\left(\mathrm{mol} / \mathrm{sec}-\mathrm{m}^{2}\right)$
$N_{\mathrm{S} \imath} \quad$ molar flux due to surface diffusion $\left(\mathrm{mol} / \mathrm{sec}-\mathrm{m}^{2}\right)$

$r \quad$ pore radius $(\mathrm{m})$

$p \quad$ relative pressure $(\mathrm{Pa})$

$P \quad$ (hydraulic) pressure $(\mathrm{Pa})$

$P_{\mathrm{A}} \quad$ permeability constant of the pure component $A\left(\mathrm{~m}^{3} / \mathrm{m}^{2}\right.$-sec-Pa-m)

$P / P_{\text {ref }}$ pressure withın the membrane/reference pressure (-)

$q$ differential heat of adsorption $(\mathrm{J} /$ $\mathrm{mol}$ )

$R \quad$ gas constant $(\mathrm{J} / \mathrm{mol}-\mathrm{K})$

$R_{\mathrm{s}} \quad$ Stokes' radius (-)

$T$ temperature $(\mathrm{K})$

$T_{\mathrm{g}} \quad$ glass-rubber transition temperature (K)

$u_{\imath} \quad$ velocity of $\iota$ in a membrane $(\mathrm{m} / \mathrm{sec})$

$v_{\mathrm{A}} \quad$ average molecular velocity $(\mathrm{m} / \mathrm{sec})$

$v_{\imath} \quad$ partial specific volume $\left(\mathrm{m}^{3} / \mathrm{kg}\right)$

$x_{i} \quad$ (molar) fraction (-)

$\alpha \quad$ selectivity (-)

$\delta \quad$ thickness (m)

$\epsilon \quad$ porosity (-)

$\eta \quad$ viscosity (Pa-sec)

$\mu_{\imath} \quad$ chemical potential, $\mu_{\imath}^{\mathrm{c}}$ is concentration dependent part of $\mu_{\imath}(\mathrm{J} / \mathrm{kg})$

$\lambda$ mean free path length of (gas) molecules $(\mathrm{m})$

$\rho \quad$ density $\left(\mathrm{kg} / \mathrm{m}^{3}\right)$

$\tau \quad$ tortuosity (-)

$\phi_{l} \quad$ volume fraction of component $l(-)$

$\Phi_{\mathrm{p}} \quad$ volume fraction of the polymer (-)

$\Phi_{s} \quad$ volume fraction of the solvent (-)

$\chi \quad$ Huggins' parameter (-)

$\Psi_{\mathrm{c}} \quad$ fraction crystallıne polymer (-)

\section{References}

1 K Keizer, R J R Uhlhorn, R J van Vuren and A J Burggraaf, Gas separation mechanisms in microporous modified $\gamma-\mathrm{Al}_{2} \mathrm{O}_{3}$ membranes, J Membrane $\mathrm{Sc} 1$, 39 (1988) 285-300 
2 J G A Bitter, Transport mechanısms in membrane separation processes, Plenum Publishing Comp , New York, NY, 1991

3 M Soltanieh and W N Gill, Reverse osmosis, Chem Eng Comm , 12 (1981) 279

4 K S Spiegler and O Kedem, Thermodynamics of hyperfiltration (reverse osmosis) criteria for efficient membranes, Desalınation, 1 (1966) 311-326

5 F, R Gilliland, R F Badour, G P Perkınson and K J Sladek, Diffusion on surfaces I Effect of concentration on the diffusivity of physically adsorbed gases, Ind Eng Chem, Fundam, 13 (1974) 95-99

6 K J Sladek, E R Gllliland and R F Badour, Diffusion on surfaces II Correlation of diffusivities of physically and chemically adsorbed species, Ind Eng Chem , Fundam , 13 (1974) 100-105

7 S Sourirajan, Reverse Osmosıs, Academıc Press, New York, NY, 1970

$8 \mathrm{G}$ Jonsson and C E Boesen, Water and solute transport through cellulose acetate reverse osmosis membranes, Desalınation, 17 (1975) 145-165

9 H K Lonsdale, U Merten and R L Riley, Transport properties of cellulose acetate osmotic membranes, $\mathrm{J}$ Appl Polym Scı, 9 (1965) 1341

10 Y M Lee, D Bourgeois and G Belfort, Sorption, diffusion and pervaporation of organics in polymer membranes, J Membrane Sc1 , 44 (1989) 161-181

11 W J Koros, A H Chan and D R Paul, Sorption and transport of various gases in polycarbonate, J Membrane $\mathrm{Scl}, 2$ (1977) 165-190, and references therein

12 J G A Bitter, Effect of crystallinity and swelling on the permeability and selectivity of polymer membranes, Desalination, 51 (1984) 19-35

13 H Strathmann, Trennung von molekularen Mischungen mit Hilfe synthetischer Membranen, Steinkopf Verlag, Darmstadt, 1979

14 E L Cussler, Membranes containing selective flakes, J Membrane Sc1, 52 (1990) 275-288

$15 \mathrm{~N}$ Wakao, J Otanı and J M Smith, AIChE J , 11 (1965) 435-438

16 R J R Uhlhorn, M H B J Huss in 't Veld, K Keizer and A J Burggraaf, High permselectivities of microporous sihca modified $\gamma$-alumina membranes, $J$ Mat Sc1 Lett , 8(10) (1989) 1135-1138

17 R J R Uhlhorn, K Keizer and A J Burggraaf, Gas and surface diffusion in modified $\gamma$-alumina systems, J Membrane Sc1 , 46 (1989) 225-241

18 R W Schofield, A G Fane and C J D Fell, Gas and vapour transport through microporous membranes I
Knudsen-Poiseulle transition, J Membrane Sc1 , 53 (1990) 159-171

19 M Yonese, H Murabayashı and H Kıshımoto, Diffusion-reaction of urea through multi-membrane containing urease Effects of micro-environments around urease and asymmetry of the membrane, J Membrane Sc1 , 54 (1990) 145-162

20 T Neplenbroek, Nitrate removal with supported liqund membranes, transport mechanism, Ph D Thesis, Univ of Twente, Enschede, 1989, Chap 2

21 R D Noble, Analysis of ion transport with fixed site carrier inembranes, J Membrane Scı , 56 (1991) 229234

22 M J D Eenink, Biodegradable hollow fibres for the controlled release of drugs II In vitro release of the contraceptive steroid levenorgestrel, $\mathrm{Ph} \mathrm{D}$ Thesı, Univ of Twente, Fnschede, 1987, Chap 7

23 H Strathmann, Electrodialysıs, in P M Bungay (Ed ), Synthetic Membranes Science, Engineering and Applications, Reidel, Dordrecht, 1986, pp 197223

24 Y Tanaka, Concentration polarization in ion exchange membrane electrodialysıs, J Membrane Scı, 57 (1991) 217-235

25 W E Katz, The electrodialysis reversal ("EDR") process, Paper presented at the International Congress on Desalınation and Water Re-use, Tokyo, Japan (1977)

26 G B van den Berg and C A Smolders, Flux decline in membrane processes, Filtration and Separation, March (1988) 115-121

27 S Nakao, T Tanabe and S Kimura, Mass transfer coefficient in ultrafiltration of macromolecular solutions, Poster presented at the 5th Int Symp on Synthetic Membranes in Science and Industry, Tubingen, Germany, September 2-5, 1986

28 J G W W Troelstra and C A Smolders, Hydrodynamic resistance of concentration polarization boundary layers in ultrafiltration, J Membrane Scı, 22 (1985) 117135

29 G B van den Berg and C A Smolders, The boundary layer resistance model for unstirred ultrafiltration A new approach , J Membrane Sc1 , 40 (1989) 149-172

30 A J Reuvers, Membrane formation diffusion induced demixing processes in ternary polymeric systems, Ph D Thesis, Univ of 'Twente, Enschede, 1987 See also $J$ Membrane Sc1, 34 (1987) 45-65 and 6786 